\title{
Analysis of the results from use of haptic peg-in-hole task for assessment in neurorehabilitation
}

\author{
Farshid Amirabdollahian ${ }^{\mathrm{a}, *}$ and Garth Johnson ${ }^{\mathrm{b}}$ \\ ${ }^{a}$ Adaptive Systems Research Group, The University of Hertfordshire, Hertfordshire, UK \\ ${ }^{\mathrm{b}}$ Centre for Rehabilitation and Engineering Studies, School of Mechanical and Systems Engineering, \\ The University of Newcastle, Newcastle, UK
}

\begin{abstract}
Haptic and robotic technologies have the potential to provide assessment during interaction with humans. This manuscript presents our earlier research during the I-Match project where a haptic peg-in-hole test was used in order to compare between healthy volunteers' performance and those with neurological impairment. Subjects all performed a series of haptic virtual peg-in-hole tasks with varying degrees of difficulty determined by the hole diameter. Haptic instrument, Phantom Desktop 1.5, allowed for recording of biomechanical data which is used to present some variant features between the two subject groups. This paper analyses the placement time, maximum peg transfer velocity, collision forces recorded during peg placement and also insertion accuracy. The first three parameters showed statistically significant differences between the two groups while the last, insertion accuracy, showed insignificant differences $(p=0.152)$. This is thought to be due to the large clearance value between the smallest hole diameter and the peg. To identify differences between the haptic peg-in-hole and the established NHPT, we are currently in process of conducting a further experiment with a haptic replica of the NHPT test, in order to investigate effects resulting from addition of haptic force feedback compared to the original NHPT test, as well as allowing to explore influences caused by the $1 \mathrm{~mm}$ clearance value as originally proposed by Wade. Furthermore, in order to investigate if this method can identify differences between subjects with different neurological conditions, a larger group of subjects with neurological conditions such as stroke, multiple sclerosis, and traumatic brain injury is required to explore potency of this approach for identifying differences between these different conditions.
\end{abstract}

Keywords: Haptics, peg-in-hole, i-match, robotic for assessment, outcome measures

\section{Introduction}

The I-Match project, funded by the European Commission under the Information Society Technologies (IST) thematic program, was a three-year project that began in November 2002. It focused on quantifying

*Corresponding author: Dr Farshid Amirabdollahian, MIET, MIEEE, Adaptive Systems Research Group, The University of Hertfordshire, School of Computer Science, College Lane, Hatfield, Hertfordshire, AL10 9AB, UK. Tel.: +44 1707286 125; Fax: +44 1707284 303. E-mail: f.amirabdollahian2@herts.ac.uk. users' upper limb performance and skills in order to aid in selecting the most suitable interface for use with his/her assistive device. The project provided a series of haptic tests, including the peg-in-hole test, aiming at distinguishing the differences seen in between healthy volunteers and subjects with neurological impairment with the longer-term objective of providing accurate and comprehensive measures of precise hand function. This approach also holds the promise of use in a tele-rehabilitation setting where the clinician may be remote from the patient. 
This paper presents the peg-in-hole haptic test and proceeds to detail the associated clinical assessment. It then analyses the results using statistical models and graphs to support the argument that biomechanical parameters identified during haptic interaction can record differences observed between different subject groups, mainly healthy volunteers versus subjects with neurological conditions.

\section{Background}

A user's ability to operate an interface is, to a major degree, dependent upon the quality of hand and arm control. While the commonest method of assessing hand control is to use one of the clinically based scales (e.g. Jebsen et al. [1], Action Research Arm Test [2]), a number of researchers have looked at more quantitative approaches. The Southampton Hand Assessment Procedure (SHAP) is an example of testing hand function in its contextual environment [3]. Using a different technology, Spyers-Ashby and colleagues [4] have used a six-degrees of freedom electromagnetic sensor to quantify upper limb tremor. In the field of robotic neurorehabilitation, the MIT-MANUS group has used haptic approaches to promote and measure upper limb function. In studies using their haptic interface, kinematic data have been used to quantify human arm movements and recovery in stroke patients [5-7]. Similarly, Reinkensmeyer et al. have used the "Arm Guide" to assess tone, spasticity and lack of coordination for patients after chronic brain injury [8]. Salazar-Torres et al. used a biomechanical device to investigate the excitability of muscle stretch reflexes in order to quantify spasticity [9]. Moreover, to assess coordination and upper limb functional state of a group of patients with different neurological disorders (e.g. Friedreich Ataxia, Parkinson's disease, Multiple Sclerosis and Muscular Dystrophy), Bardorfer et al. have used a PHANToM haptic interface and a virtual labyrinth [10]. It is acknowledged that the aforementioned studies represent only a small number of the existing and ever-growing research aiming to quantify upper limb function.

\subsection{The haptic peg-in-hole test}

The peg-in-hole task consists of two haptically rendered holes and a cylindrical peg, which is to be alternately inserted in each hole. Figure 1 shows the

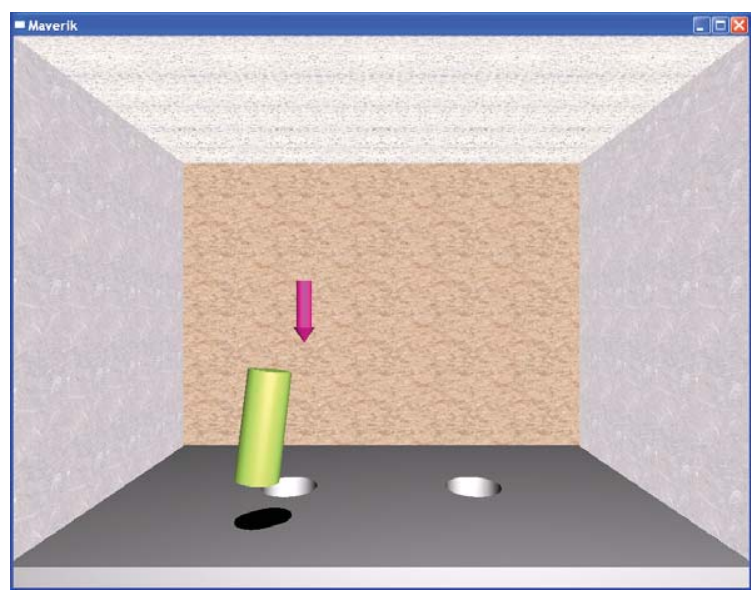

Fig. 1. Visual (on screen) representation of the peg-in-hole task.

graphical user interface presented for this task, correlating with dimensions of the haptically rendered model.

The test is inspired by the validated and established Nine-Hole-Peg-Test (NHPT) used in clinical assessment. The NHPT requires inserting nine pegs in holes arranged in three rows and three columns. The completion time or the number of pegs inserted in a given time is seen as a reliable measure of subject's skills and performance [11]. As seen in Fig. 1, the haptic peg-inhole test provides only two holes and uses only one peg during any one session. It is intentionally designed to be different from the conventional test as it provides a chance to assess many other aspects of the performance, for example: it is hypothesized that repetitive movements to the same positions allow for investigating both repeatability and extent of learning to perform better; with a two-hole-peg-test where subjects either perform a left-to-right or a right-to-left half-cycle, it is possible to compare performance variations towards and away from the dominant hand, while due to the freedom given in choosing the pegs in the conventional NHPT, this is not possible; furthermore the two hole setting also provides a simple base for comparing the performance using the conventional Fitt's model for motor control [12].

Haptic presentation of the virtual world is created using the PHANToM desktop 1.5 haptic interface from SensAble Technologies, USA (www.sensable.com). Being developed as a virtual world, the peg-in-hole test also allows for altering experimental parameters such as: peg diameter, peg height, peg weight, hole diameter, 


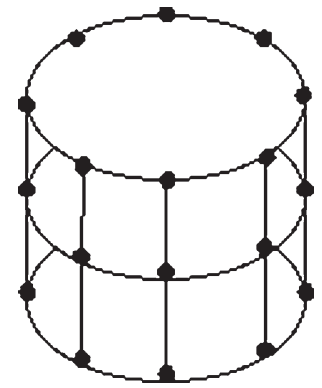

Fig. 2. Slicing and sectioning the peg for haptic collision detection.

separation distance between holes and clearance (peg vs. hole). The peg collision with the holes and the other solid surfaces such as walls shown by Fig. 1 is detected by detecting collision between these objects and slices of the peg, which are sectioned as shown by dots in Fig. 2. Using a novel mathematical collision detection algorithm, multiple contacts between the peg, the table and holes are detected and haptic feedback is produced upon collisions.

\section{Experimental settings and procedures}

The PHANToM offers 3 active and 3 passive degrees of freedom. The orientation of the virtual peg mimicked that of the PHANToM stylus in real-time. The virtual table was placed on a horizontal plane as presented in Fig. 1. Shadow cues were used to provide better depth perception.

The virtual table created for this experiment presented the holes with $150 \mathrm{~mm}$ separation distance between them. The hole diameter (HD) varied between $80 \mathrm{~mm}, 60 \mathrm{~mm}$ or $40 \mathrm{~mm}$ resulting in three distinct experimental settings. The peg diameter was $30 \mathrm{~mm}$ leaving a $10 \mathrm{~mm}$ clearance between the peg and the smallest hole.

Participants ranged from healthy volunteers (HV) to subjects recovering from multiple sclerosis (MS), stroke (S) or traumatic brain injury (TBI). Subjects with neurological impairment were patients at the Hunters Moor hospital, Newcastle upon Tyne. Fifty three subjects participated in the trial. Subjects had no prior knowledge of test objectives and peg-in-hole was presented as a potential robotic exercise, which could influence recovery. All subjects gave informed consent to participate and could leave the experiment at any point. From these, 41 subjects completed the experiment and their demographic details are presented in Table 1. These resulted in four subject groups: HV, MS, $\mathrm{S}$ and TBI. Due to the difficulty of matching subject numbers between the three groups with neurological impairments (MS $(n=14), \mathrm{S}(n=2)$ and TBI $(n=2))$, and given that main objective of this study was to investigate differences between healthy subjects and those with neurological conditions, the three groups are merged as neurological group in this study.

Subject sat comfortably in front of the PHANToM desktop device as shown in Fig. 3. They were instructed to insert the peg into the left hole, remove it and insert it into the right hole (starting from mid-position). This was to be performed 20 times ( 10 cycles) before moving to the next experimental setting. They were instructed to move as quickly as possible while trying to minimise the collision with the table and the walls of the holes during peg placement and removal. The visual and auditory cues were also explained. The peg insertion and removal was accompanied by a downward or upward guiding arrow presented on the screen

Table 1

Subject demographic table

\begin{tabular}{lcccccc}
\hline & Total & Male & Female & $\begin{array}{c}\text { Left } \\
\text { hand }\end{array}$ & $\begin{array}{c}\text { Right } \\
\text { hand }\end{array}$ & Age \\
\hline Healthy & 23 & 6 & 17 & 2 & 21 & $38 \pm 15$ \\
Neurological & 18 & 8 & 10 & 4 & 14 & $51 \pm 12$ \\
Total & 41 & 14 & 27 & 6 & 35 & $44 \pm 15$ \\
\hline
\end{tabular}

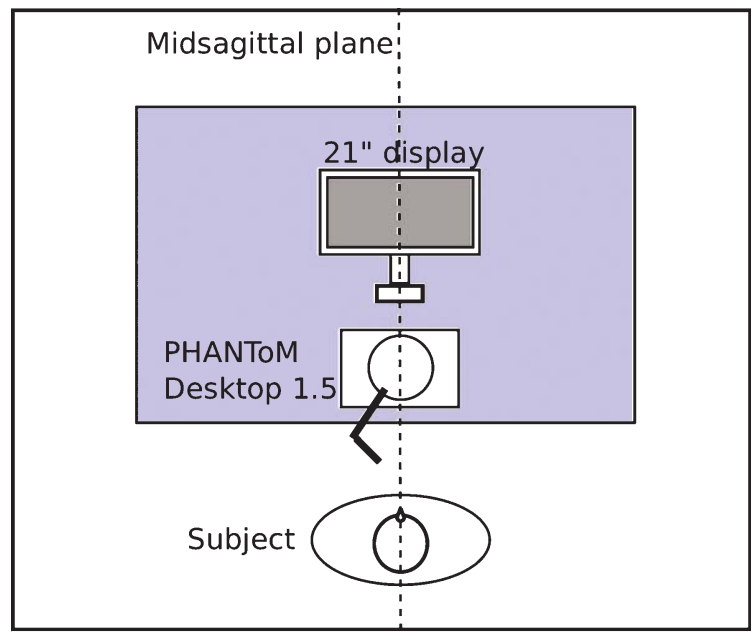

Top view of experimental setup

Fig. 3. Experimental setup. 
(shown in Fig. 1). A loud audio beep was also played once at each successful peg insertion.

\section{Results and analysis}

Data were logged at an average sampling frequency of $1000 \mathrm{~Hz}$. Position, Orientation, Velocity and Contact/Collision reaction Forces were recorded as vectors in Cartesian coordinate frame ( $x, y$ and $z$ attributes) with SI units. During the data logging, data were coded with tags allowing easy selection of relevant data related to each half-cycle. In order to investigate differences between repetitions, five full cycles were selected (cycles 4 to 9) for each of the 3 settings. Figure 4 shows a 3D presentation of one typical session during the experiment produced using MATLAB.
The following parameters were chosen for analysis here: the time taken to complete each full cycle, time of left-to-right (L2R) cycles, time of right-to-left (R2L) cycles, collision forces during each half cycle, insertion error and maximum velocity during peg transfer.

\subsection{Analysis method}

PASW 18.0 was used to analyse the results statistically. Figure 5 shows the study subject groups, experimental settings and the process followed during the data analysis. As there were 5 selected half cycles for each $\mathrm{L} 2 \mathrm{R}$ and $\mathrm{R} 2 \mathrm{~L}$ peg placement, a repeated measures ANOVA was first used to identify differences between recorded parameters for these repetitions. All four parameters showed insignificant differences for these repetitions ( $p$-value $>0.05$ ).

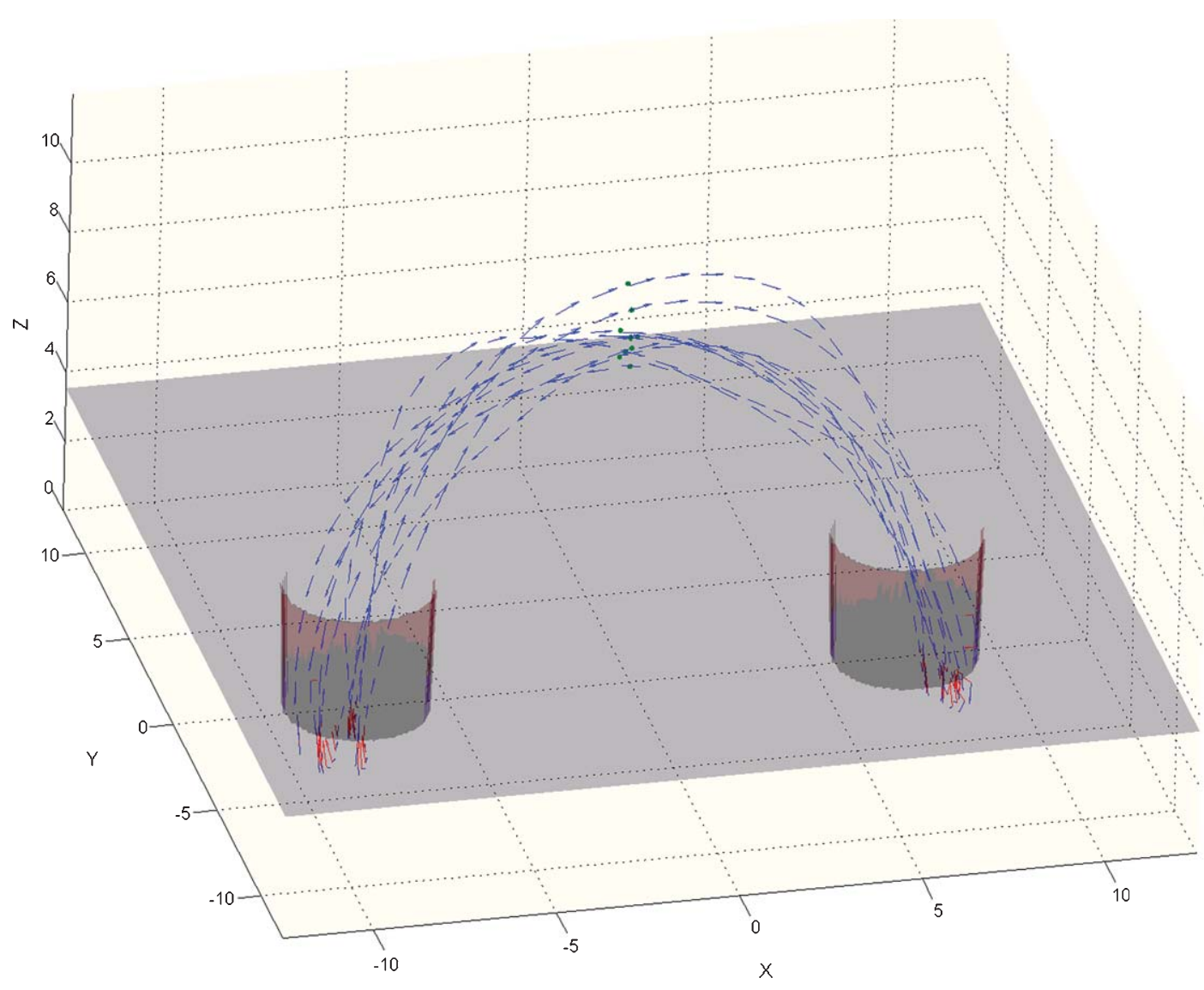

Fig. 4. 3D representation of performance by one of the subjects, analysed using MATLAB. 


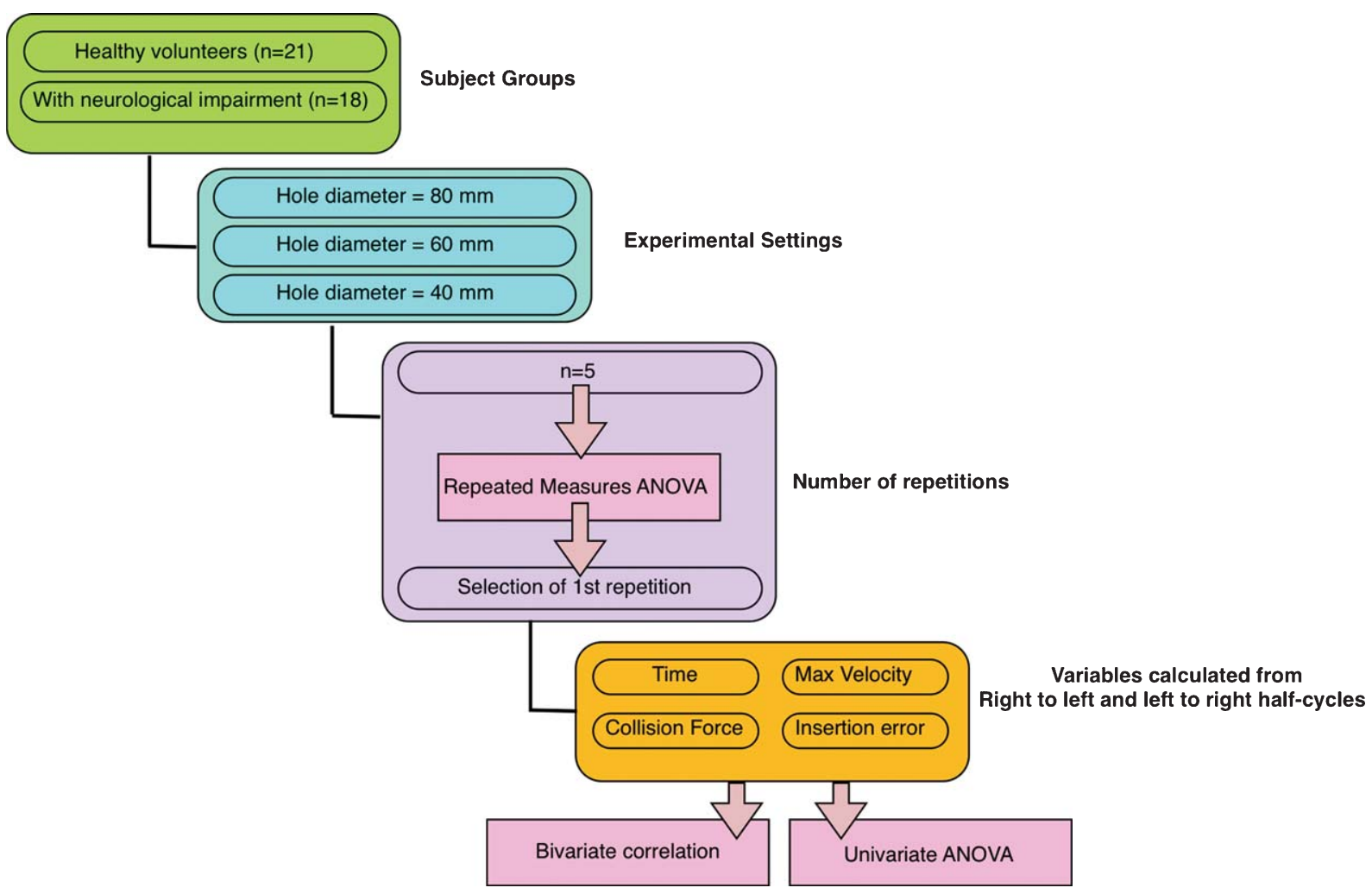

Fig. 5. Study setup versus gathered and analysed parameters.

Due to this invariance, a Univariate model was then used to analyse the data from the 1 st repetition for $\mathrm{L} 2 \mathrm{R}$ and R2L half cycles.

\subsection{Analysis of movement time}

Due to insignificant differences between repetitions, only the first occurrences of L2R and R2L peg placement were analysed. Sample data for one subject is provided in Table 2. The Univariate model used movement time as its parameter while using subject group ( $1=$ Neurological Group, $2=$ Healthy Group), hole diameter $(4,6$ and $8 \mathrm{~cm})$, and trajectory direc- tion $(1=\mathrm{R} 2 \mathrm{~L}, 2=\mathrm{L} 2 \mathrm{R})$ as its fixed factors. The results showed highly significant differences between the subject groups ( $p$-value $<0.0005$ ) while failing to identify significant differences based on hole diameter or trajectory direction. Figure 6 presents a graph comparing between the two subject groups, different hole diameters and trajectory directions.

\subsection{Analysis of collision forces}

Collision forces were recorded during interaction with the haptic holes, the table and the walls. During

Table 2

Sample data from one subject, one R2L and L2R half cycles

\begin{tabular}{lccccccccc}
\hline Subject & Session & HD & $\begin{array}{c}\text { Subject } \\
\text { group }\end{array}$ & $\begin{array}{c}\text { L2R/ } \\
\text { R2L }\end{array}$ & Repetition & Time & $\begin{array}{c}\text { Force } \\
\begin{array}{c}\text { Max } \\
\text { velocity }\end{array}\end{array} \begin{array}{c}\text { Insertion } \\
\text { error }\end{array}$ \\
\hline 2 & 5 & 8 & 1 & 2 & 1 & 2.7 & 14.55 & 267.34 & 2.19 \\
2 & 7 & 6 & 1 & 2 & 1 & 3.43 & 83.68 & 297.26 & 1.18 \\
2 & 8 & 4 & 1 & 2 & 1 & 4.17 & 66.35 & 285.77 & 1.13 \\
2 & 5 & 8 & 1 & 1 & 1 & 2.93 & 16.84 & 366.65 & 1.66 \\
2 & 7 & 6 & 1 & 1 & 1 & 5.12 & 88.94 & 257.16 & 0.47 \\
2 & 8 & 4 & 1 & 1 & 1 & 4.45 & 49.69 & 281.97 & 1.32 \\
\hline
\end{tabular}




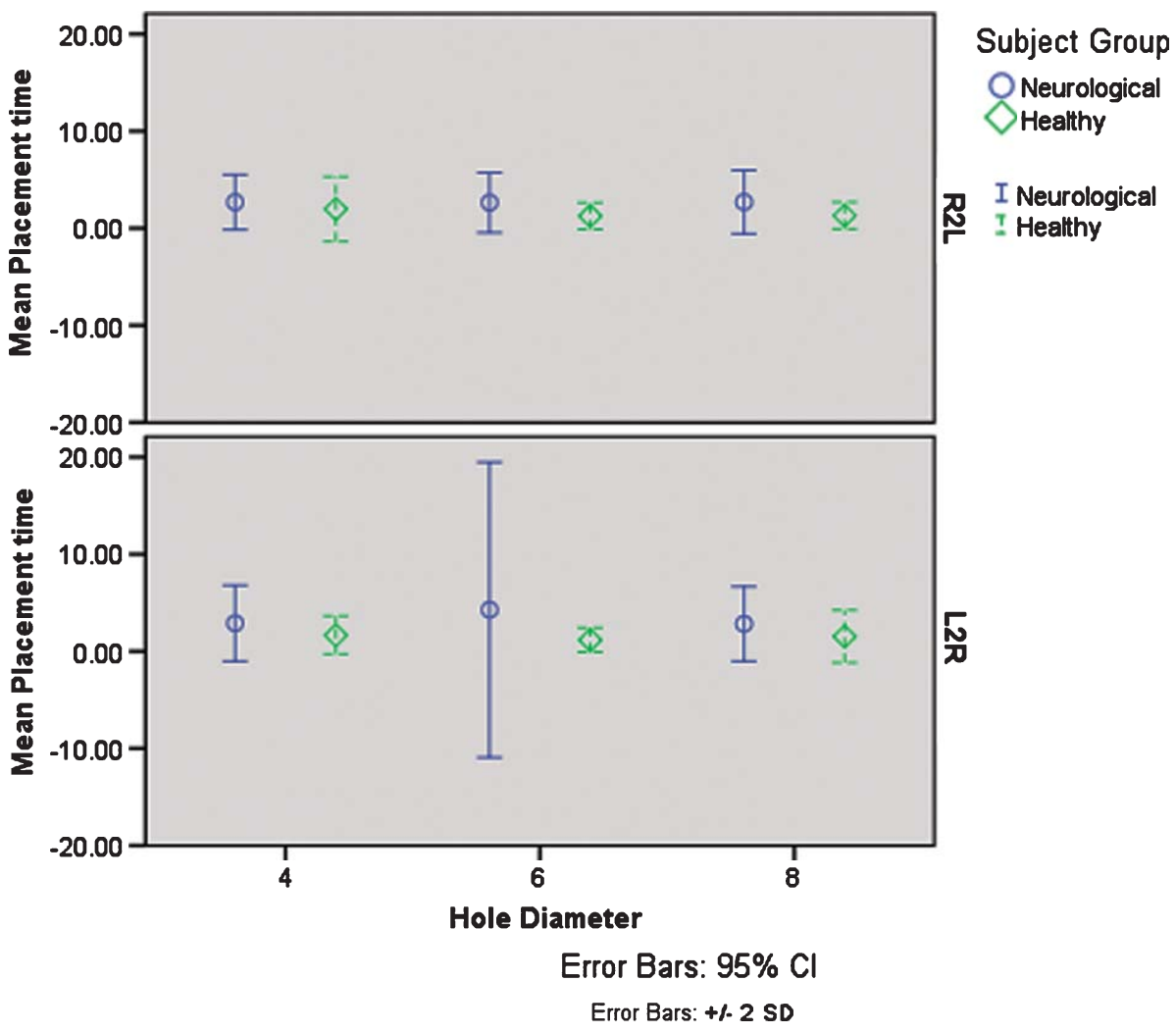

Fig. 6. Comparing placement time for R2L and $\mathrm{L} 2 \mathrm{R}$ half cycles and subject groups.

interaction, such collisions were detected and interaction forces calculated by the PHANToM device and recorded using the peg-in-hole program. For each halfcycle, these forces were summed to produce a Collision Force Vector, used to calculate a resultant collision force magnitude for this analysis.

Similar to the movement time, collision force magnitudes were analysed using the Univariate Model. The same parameters were used for this analysis and the results showed a strong effect for subject group ( $p$ value $=0.016)$ and interaction between subject group and hole diameter $(p$-value $=0.017)$. These differences are visually presented by Fig. 7 .

\subsection{Analysis of maximum transfer velocity}

During the interaction, transfer velocity is logged as a vector in the Cartesian coordinate frame. The maximum velocity magnitude (MaxVM), was then found by a search algorithm during each L2R or
R2L half-cycle. This MaxVM was analysed using the Univariate model with the same parameters as mentioned earlier. The results showed significant differences between the two groups ( $p$-value <0.0005) but failed to highlight differences between L2R and R2L half-cycles, or between different hole-diameters (Fig. 8).

\subsection{Analysis of the insertion error}

An insertion error was defined as the distance between the centre of the peg and the centre of the hole during each insertion into the right or left hole. As a 2D parameter, this was calculated on the horizontal plan passing the table surface. Figure 9 presents the spread of this error for a single subject where it is clear that this point is not always close to the centre of the hole. This parameter was also analysed to compare between the two groups, different hole diameters and trajectory directions. The results (Fig. 10) 


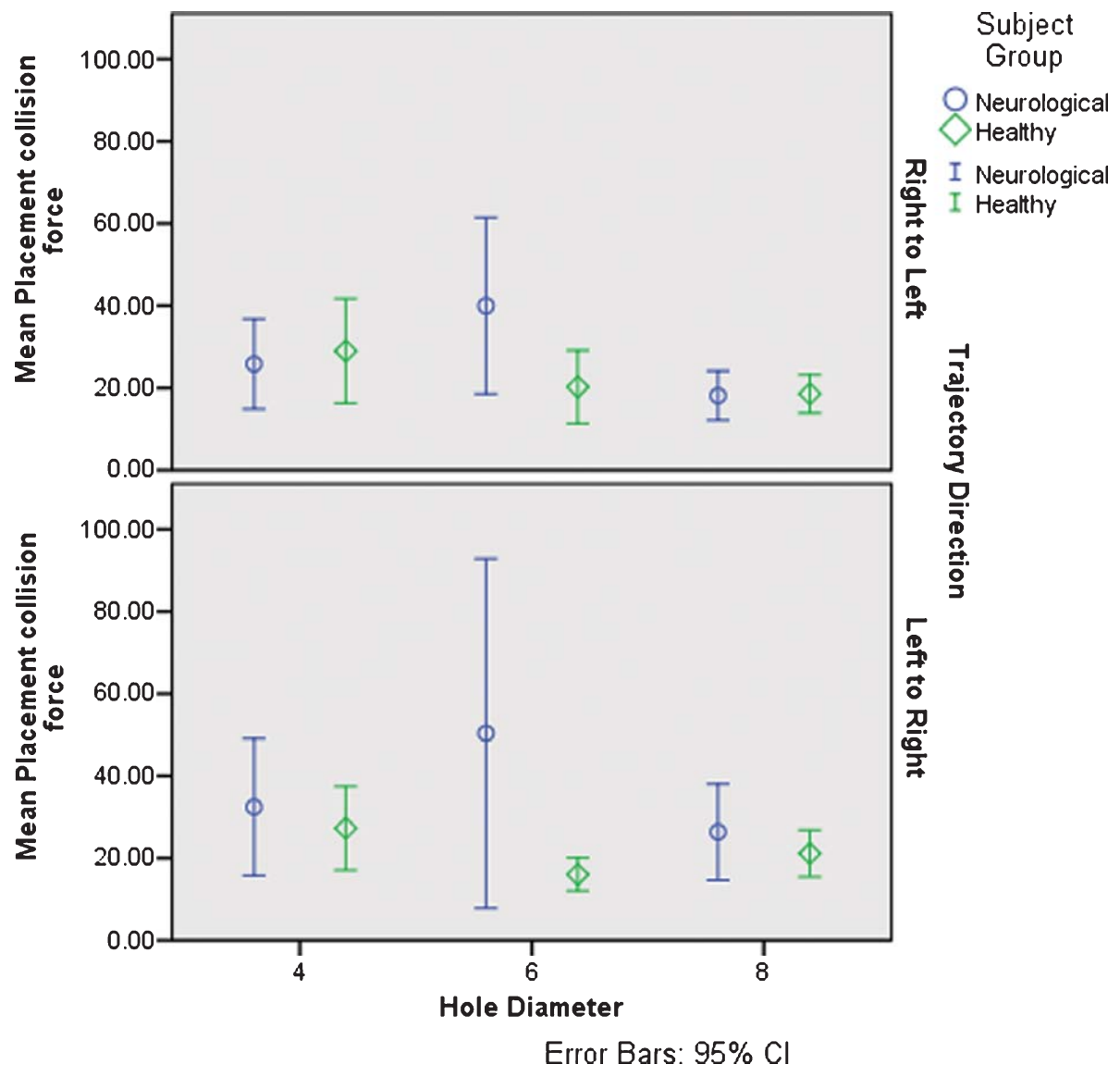

Fig. 7. Comparing collision forces for R2L and L2R half cycles and subject groups.

show insignificant differences between the two groups ( $p$-value $=0.152)$ while highlighting significant differences between insertions for different hole diameters $(p$-value $<0.0005)$.

\subsection{Bivariate-correlation between factors}

A final step taken was to analyse the bivariate correlations between the four factors mentioned previously. This procedure was used to compute Pearson's correlation coefficient and its statistical significance. The results obtained from the correlation analysis showed that there were significant correlations between movement time and collision forces (Pearson's correlation coefficient: 0.663 , significance: $p$-value $<0.0005$ ); and movement time and maximum transfer velocity (Pearson's correlation coefficient: -0.212 , significance: $p$-value $=0.001)$. However, there was no significant correlation between movement time and insertion error (Pearson's correlation coefficient: 0.041, significance: $p$-value $=0.522$ ), while insertion error was correlated with both placement collision force (Pearson's correlation coefficient: 0.195 , significance: $p$-value $=0.002$ ) and maximum transfer velocity (Pearson's correlation coefficient: 0.212 , significance: $p$-value $=0.001)$. Collision forces and maximum transfer velocity were also correlated (Pearson's correlation coefficient: 0.352, significance: $p$-value $<0.0005)$.

\section{Discussions}

A major purpose of this initial study has been to identify measurement parameters, which are likely to be of clinical relevance when measuring rehabilitation progress. Comparison of performance time between 


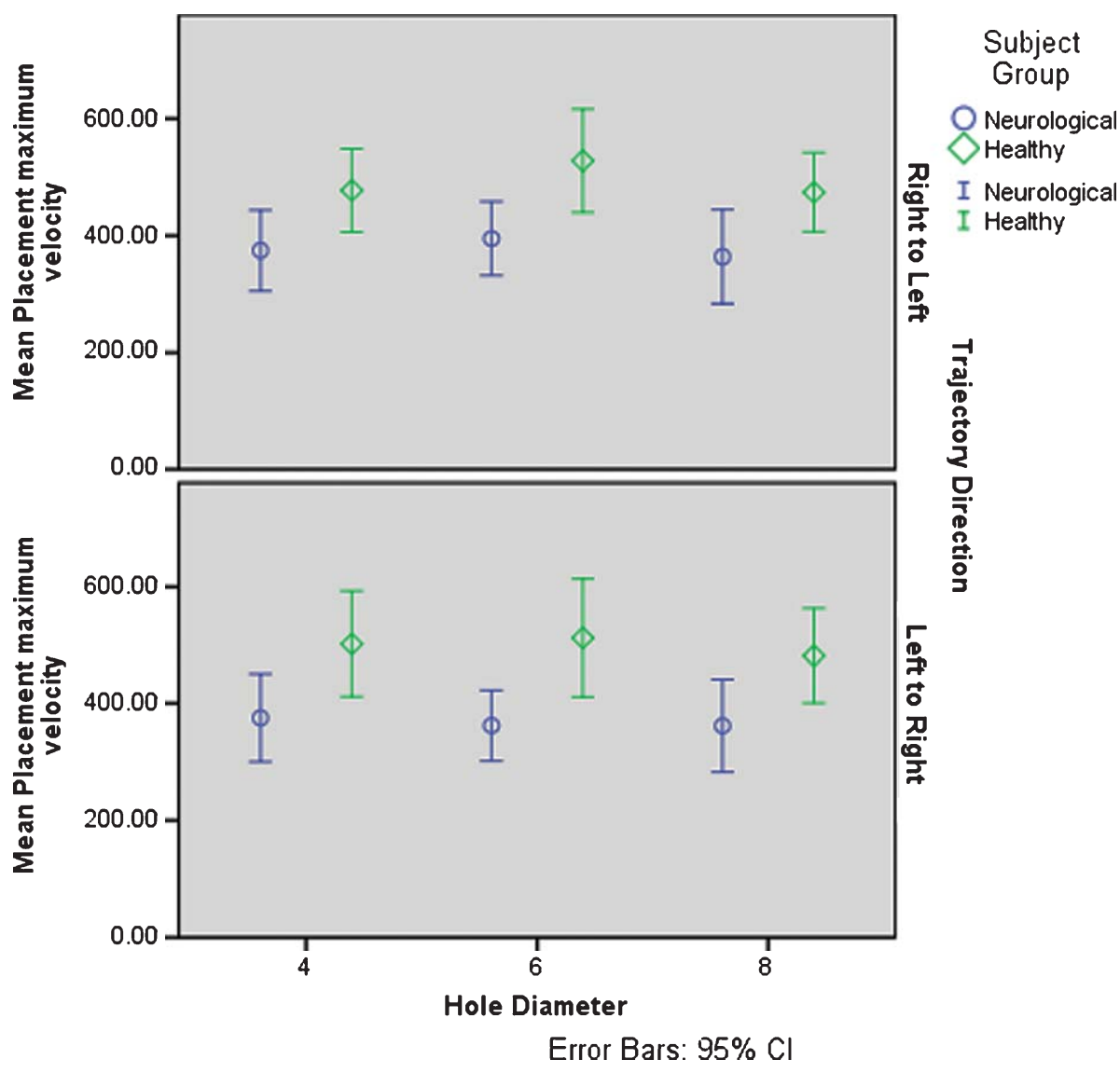

Fig. 8. Comparing maximum velocity for R2L and L2R half cycles and subject groups.

different subject groups showed significant differences between the unimpaired and patient groups. This trend was further confirmed by results from collision force and maximum velocity. However, insertion error failed to support significant differences between the two subject groups. This finding is probably not surprising since the test provides no visual or auditory feedback of insertion accuracy. Since the clearances are relatively large, it is fairly easy for the subject to avoid collision with the side of the hole with a small level of precision. This is further supported by the evidence from Pearson's correlation that movement time was significantly correlated with maximum velocity magnitude and collision force but not with the insertion error.

As might be expected, insertion error showed strong effects for the hole diameter, but this was not significantly different between the two groups. Moreover, collision force failed to show strong evidence for differences related to hole diameter again suggest- ing that the relatively large clearance allowed easy insertion.

Upon studying Figs 6 and 7, it is clear that L2R portion of the movement time and collision force both present large variations for a $60 \mathrm{~mm}$ diameter hole and the neurological subject group. However, neither of the Univariate models have identified subject group interaction with trajectory direction as a significant interaction.

This study has presented proof of concept ideas where a clinically established and validated test such as NHPT can be replicated using the haptic simulation. Although movement time here was calculated in a different manner from the traditional test, it was still capable of distinguishing differences between different subjects and subject groups. Similarly, collision force and maximum velocity magnitude showed potential for identifying differences between the two subject groups. 


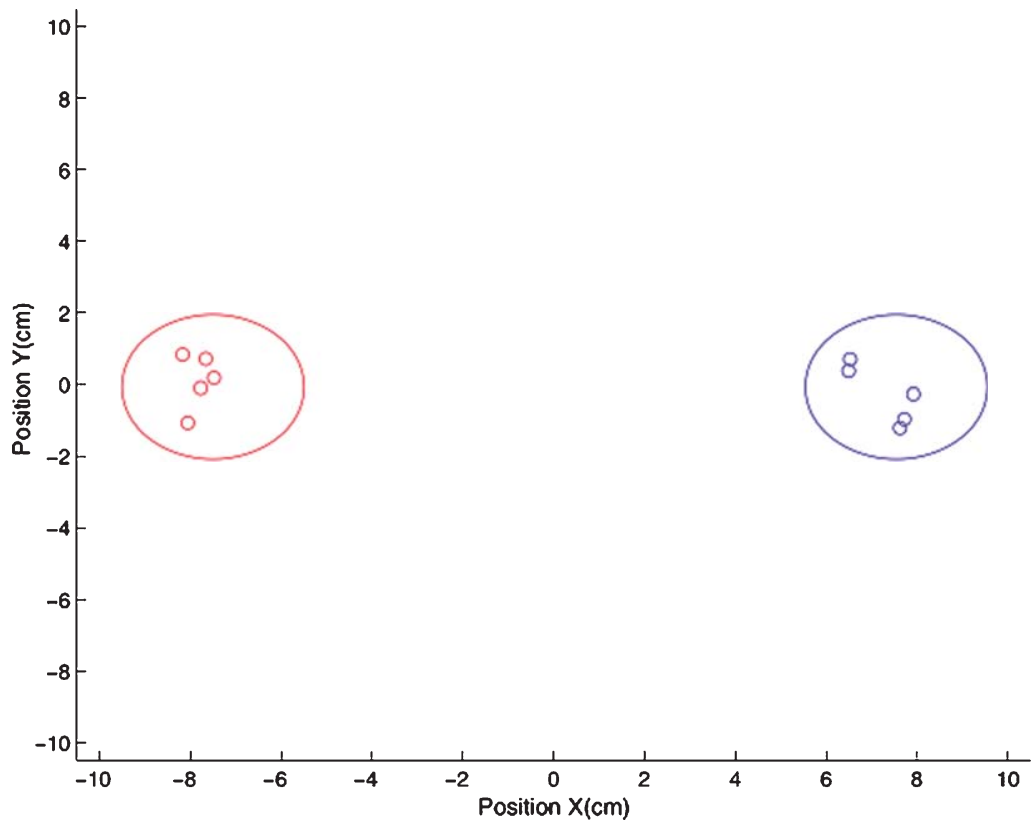

Fig. 9. Insertion point at left and right holes $(\mathrm{z}=0$, table level).

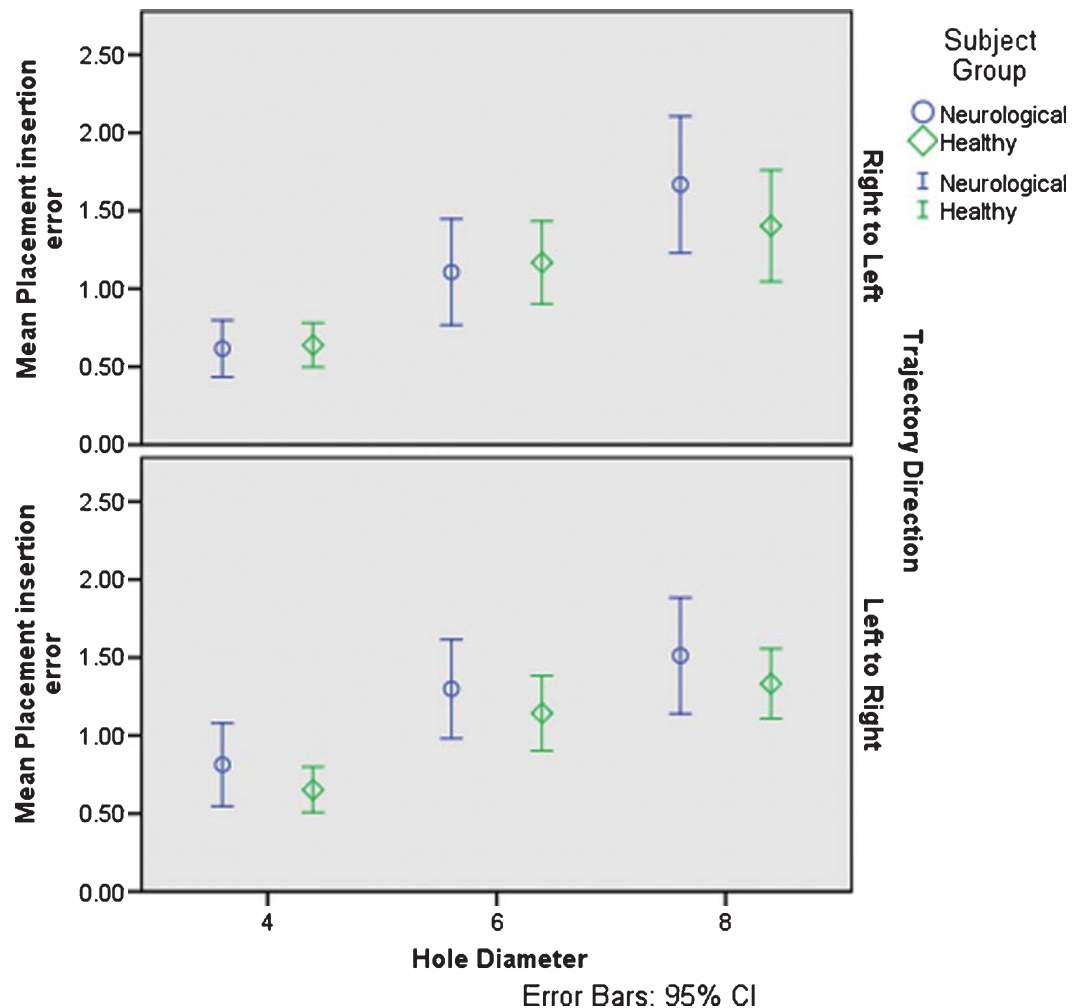

Fig. 10. Comparing insertion error for $\mathrm{R} 2 \mathrm{~L}$ and $\mathrm{L} 2 \mathrm{R}$ half cycles and subject groups. 
There are clearly some challenges for future development. While the original NHPT used a $1 \mathrm{~mm}$ clearance between the peg and the hole diameter, the $10 \mathrm{~mm}$ clearance used here is thought to have not posed significant challenge illustrated by insignificant differences between the collision forces for different hole diameters.

\section{Conclusions}

This paper has presented a novel haptic test for analysing performance through interaction. By replicating and modifying one of the established clinical measures into a haptic assessment, it has shown that it is possible to identify differences between the unimpaired group and subjects with neurological conditions. This was evident from movement time, collision force and maximum velocity magnitude during peg transfer. The failure to demonstrate differences in insertion error is attributed to the use of large clearances and the absence of feedback for insertion accuracy.

While the study has shown the ability to distinguish between normal subjects and those with impairment, the small numbers of subjects with different neurological conditions made it impossible to investigate differences between different pathologies.

Further development and testing are required before proposing this test as a suitable replacement for the traditional Nine-Hole-Peg-Test (NHPT). The major requirements are further development of the system to allow smaller clearances and improved user feedback followed by larger scale clinical studies allowing validation and calibration of this new tool against the traditional NHPT and other relevant clinical measures. Further work in this area is currently being carried out to achieve smaller peg clearances and so a closer replication of the NHPT. In conclusion, the authors believe that rapid developments in haptic technology hold the promise of higher technology and low cost systems capable of detailed measurement of precise hand function and motor control.

\section{Acknowledgements}

We are grateful to all our colleagues in the $i$-match consortium (University of Newcastle upon Tyne, UK; University of Ljubljana, Slovenia; HITEC SNT con- sultants, Greece; Trinity College Dublin, Ireland; and Fondazione Don Gnocchi (S.I.V.A.)), Italy, for their ongoing commitment to this work.

We are also grateful to staff at Hunters Moor hospital in Newcastle and subjects that agreed to participate in this study.

Last but not least, we are thankful to Mr Germano Gomes who had contributed to the development of pegin-hole and its MATLAB analytical procedure from the start of the project to its end.

\section{References}

[1] R.H. Jebsen, N. Taylor and R.B. Trieschmann, An objective and standardised test of hand function, Arch Phys Med Re habil 50 (1969), 311-319.

[2] R.C. Lyle, A performance test for assessment of upper limb function in physical rehabilitation treatment and research, International Journal of Rehabilitation Research 4 (1981), 483-492.

[3] C.M. Light, P.H. Chappell and P.J. Kyberd, Establishing a standardized clinical assessment tool of pathologic and prosthetic hand function: Normative data, reliability, and validity, Archives of Physical Medicine and Rehabilitation 83(6) (2002), 776-783

[4] J.M. Spyers-Ashby, M.J. Stokes, P.G. Bain and S.J. Roberts, Classification of normal and pathological tremors using a multidimensional electromagnetic system, Medical Engineering and Physics 21(10) (1999), 713-723.

[5] H.I. Krebs, M.L. Aisen, B.T. Volpe and N. Hogan, Quantization of continuous arm movements in humans with brain injury, in Proceedings of the National Academy of Sciences of the United States of America 1999, pp. 46454649.

[6] H.I. Krebs, B.T. Volpe, M.L. Aisen and N. Hogan, Increasing productivity and quality of care: Robot-aided neuro-rehabilitation, Journal of Rehabilitation Research and Development 37(6) (2000), 639-652.

[7] H.I. Krebs, N. Hogan, M.L. Aisen and B.T. Volpe, Robotaided neurorehabilitation, Ieee Transactions on Rehabilitation Engineering 6(1) (1998), 75-87.

[8] D.J. Reinkensmeyer, L.E. Kahn, M. Averbuch, A. McKennaCole, B.D. Schmit and W.Z. Rymer, Understanding and treating arm movement impairment after chronic brain injury: Progress with the ARM guide, Journal of Rehabilitation Research and Development 37(6) (2000), 653662.

[9] J. Salazar-Torres, A.D. Pandyan, C.I.M. Price, R.I. Davidson, M.P. Barnes and G.R. Johnson, Does spasticity result from hyperactive stretch reflexes? Preliminary findings from a stretch reflex characterization study, Disability and Rehabilitation 26(12) (2004), 756-760.

[10] A. Bardorfer, M. Munih, A. Zupan and A. Primozic, Upper limb motion analysis using haptic interface, Ieee Asme Transactions on Mechatronics 6(3) (2001), 253-260.

[11] D.T. Wade, Measurement in Neurological Rehabilitation, Oxford University Press, Oxford, England, 1992, p. 408. 
[12] P.M. Fitts, The information capacity of the human motor system in controlling the amplitude of movement, Journal of Experimental Psychology 47 (1954), 381-391.

[13] I.S. MacKenzie, Movement time prediction in humancomputer interfaces, in Reading in Human-Computer Interaction, R.M. Baecker, et al., eds, CA, Kaufmann, 1995, pp. 483-493.
[14] I.S. MacKenzie and W. Buxton, Extending Fitts' law to twodimensional tasks, Proceedings of the SIGCHI Conference on Human Factors in Computing Systems, ACM, New York, NY, USA, 1992, pp. 219-226.

[15] A. Murata and H. Iwase, Extending fitts' law to a threedimensional pointing task, Human Movement Science 20(6) (2001), 791-805 

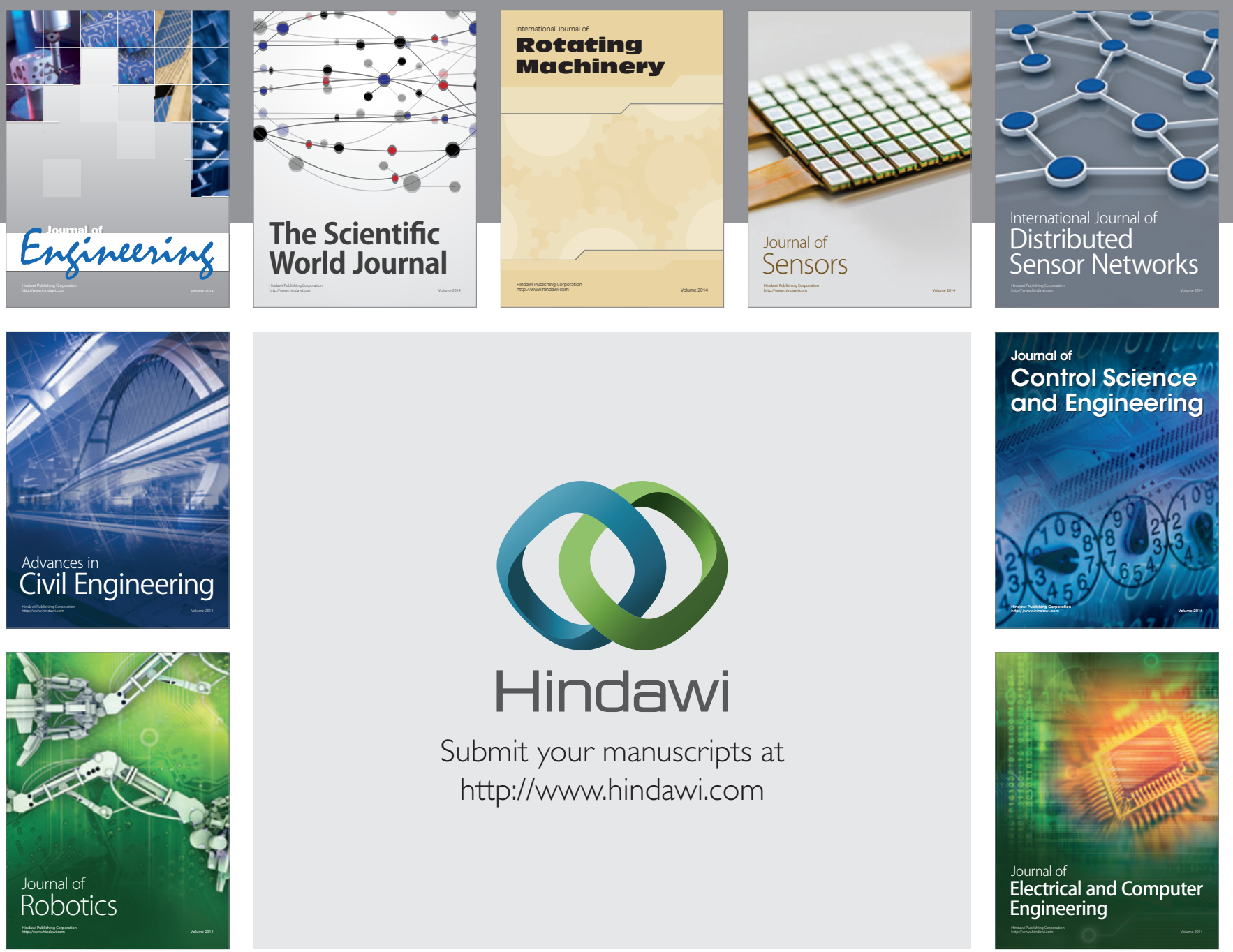

Submit your manuscripts at

http://www.hindawi.com
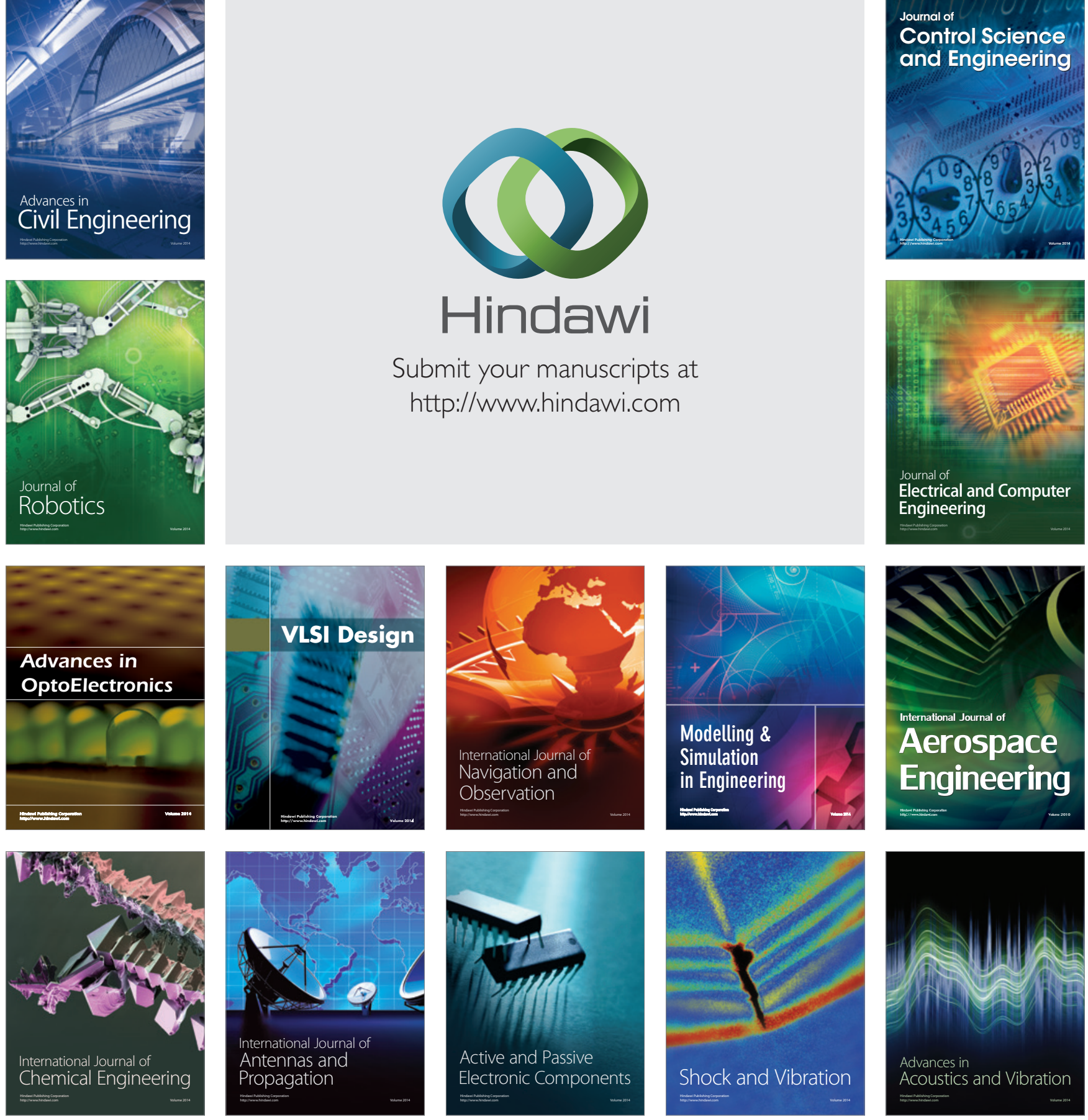